\title{
Suddenly developed hemorrhagic olecranon bursitis releated to traumatic asymptomatic heterotopic ossification
}

\author{
Zeliha Unlu, Yavuz Selim Tokmak and Ali Sahillioglu \\ Department of Physical Medicine and Rehabilitation, Celal Bayar University, Manisa, Turkey
}

\begin{abstract}
Hemorrhagic olecranon bursitis is seen rarely. In this case, hemorrhagic olecranon bursitis developed at the asymptomatic side of the patient who had reflex sympathetic dystrophy developed secondary to traumatic amputation of the right hand fingers. Heterotopic ossification which induced from trauma detected after the clinical examination at the asymptomatic side. The presentation of the case which was considered as hemorrhagic olecranon bursitis related to traumatic asymptomatic Heterotopic ossification was aimed. The developing of hemorrhagic olecranon bursitis which considered as a local complication of Heterotopic ossification will provide a different perspective for similar cases.
\end{abstract}

\section{Introduction}

As the olecranon bursitis is a pathology which is often seen in rheumatology practice, in etiology, rheumatoid arthritis, crystal arthropaties, pigmented villonodular synovitis, trauma, olecranon spur, avulsion fractures, myeloproliferative diseases from systemic inflammatory diseases and infection may be the responsible reasons [1]. Risk factors are including drug utilization, diabetes mellitus, azotemia (uremia), alcohol, oral anticoagulant drugs and long-term steroid utilization. Clinical findings such as oedema, increasing in temperature, pain and pain-related disability in joint mobility are identified on elbow area. HOB is relatively less often and generally having non-inflammatory features [2-5]. Etiologic reasons of HOB are similar with the above reasons. There is not enough data in literature about the relationship between calcified reasons which are mentioned in aetiology like olecranon spur and crystal arthropathies and HOB.

$\mathrm{HO}$ is a complication which can occur frequently after a trauma, especially on elbow area. It often occurs in neurological deficit cases which are related with head trauma or spinal cord injuries [5-7]. There is not enough data about the bursitis from the secondary complications occur around the tissue with HO. In this case report the aim is to attention on the $\mathrm{HOB}$ which is triggered by silent $\mathrm{HO}$ occurs after the trauma.

\section{Case report}

On a 75 years old male patient, right brachial artery injury, amputation onwards 4th and 5th right hand finger distal phalanx and stabile fractures of radius and olecranon on right elbow occurred on a motor vehicle crash 2 months before he was hospitalised in our clinic. Patient had no drug utilization past and another systemic disease. Brachial artery repairment has been performed to the patient and right elbow has been immobilized without any orthopaedic interference. Patient was reported diameter increment on right elbow (about $2 \mathrm{~cm}$ to the wrist, and $5 \mathrm{~cm}$ on hand level), redness, decreasing in pilosity, increasing of temperature, allodini, numbness in the hand, decreasing of joint mobility on elbow-hand-wrist and shoulder pain in the right upper extremity when he applied to our clinic.
Thyroid, liver and kidney tests of the patient were detected in normal limits. As the rates were: C-reactive protein $0.46 \mathrm{mg} /$ dl, leucocyte: 5200, hemoglobin 13:5 g/dl, sedimentation: $31 \mathrm{~mm} /$ hour, skelethal alkalyne phosphatase:6.2, calcium oxalate crystal has been detected during urinalysis. In hand graphy, patchy osteopaenia and amputations has been detected. In right elbow lateral graphy, traumatic avulsion of bone accompanying with the myositis ossificans and calcified changes is triceps tendon was detected. In right elbow MR, similar with graphy, calcified tendinitis in triceps tendon and myositis ossificans on distal surface of humerus was detected. The case was hospitalized with RSD diagnosis. For RSD, right upper extremity ROM and strengthening exercises, superficial lymphedema massage, shoulder-hand conventional TENS were performed for 45 minutes. Stellar ganglion block with scopy was done to the patient 3 times once a week. After 15 seasons of physical therapy and 3 stellar ganglion blocks, circumference measurements were decreased $3 \mathrm{~cm}$ on hand and $2 \mathrm{~cm}$ on the midpoint elbow-hand area than before the therapy and also complaints were decreased significantly.

On the 3rd day of the physical therapy, swelling, increase of temperature and minimal redness on left elbow has suddenly and spontaneously occurred on olecranon. Range of motion and systemic findings of patient, including the fever, was normal. Olecranon bursitis is thought in diagnosis with these findings. In laboratory investigations, $\mathrm{RF}$, anti CCP was at normal levels and uric acid was determined as 4.2. $10 \mathrm{cc}$ haemorrhagic looking synovial liquid was aspirated with ultrasonography guide. Synovial liquid culture was negative and there was not any pathological finding for crystal arthropathy or pigmented villonodular synovitis. WBC: 500, RBC: 50000, PLT:16000 were

Correspondence to: Ali Sahillioglu, Department of Physical Medicine and Rehabilitation, Celal Bayar University, Manisa, Turkey, E-mail: dralisahillioglu@ gmail.com

Key words: hemorrhagic olecranon bursitis, heterotopic ossification, reflex sympathetic dystrophy

Received: May 23, 2017; Accepted: June 20, 2017; Published: June 23, 2017 
detected in aspiration material and aseptic HOB diagnosis was decided. Traumatic osteochondral bone lesions and ossifications at soft tissue (HO) were detected on elbow x-ray and MRI imaging. Olecranon bursitis was detected too on MR imaging at the left elbow (Figures 1a and $1 \mathrm{~b})$.

Bursitis on the left elbow of the patient was resolved rapidly after the aspiration of fluid and the swelling was minimal when he discharged. RSD on the right upper limb and left olecranon bursitis was completely resolved at first month visit.

\section{Discussion}

It was considered that $\mathrm{HOB}$ was secondary to the missed trauma which was on the other upper extremity. Ossified lesions and bone disarrangement on the radius head at the HOB -side which was detected on radiographs and MRI imaging was interpreted as HOinduced trauma. In the literature, Strickland et al. [1] found HOB in a patient with myeloproliferative disorder and drew attention on an association with inflammatory disease in other patients. We did not found any information about the association between the $\mathrm{HO}$ and $\mathrm{HOB}$ in literature search. Indeed, pseudogout and olecranon spur relationship between $\mathrm{HOB}$ suggests that calcified formations may play

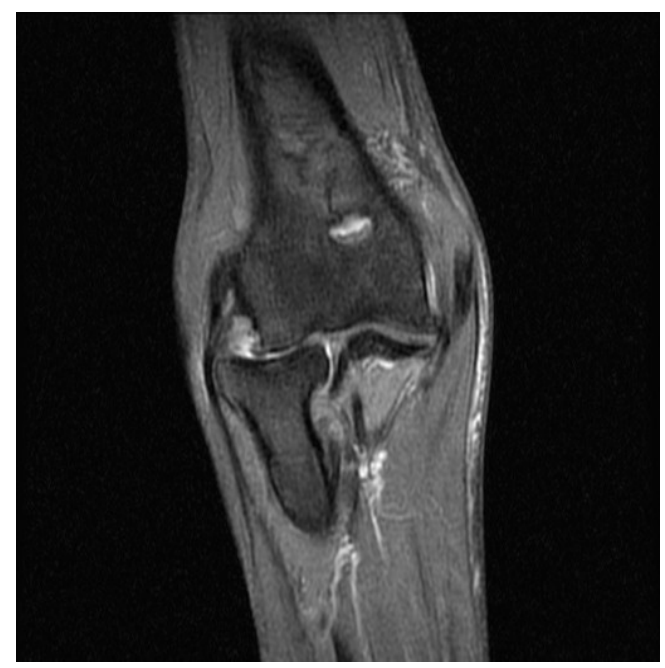

Figure 1a. Left Elbow Cor PD FSat MR

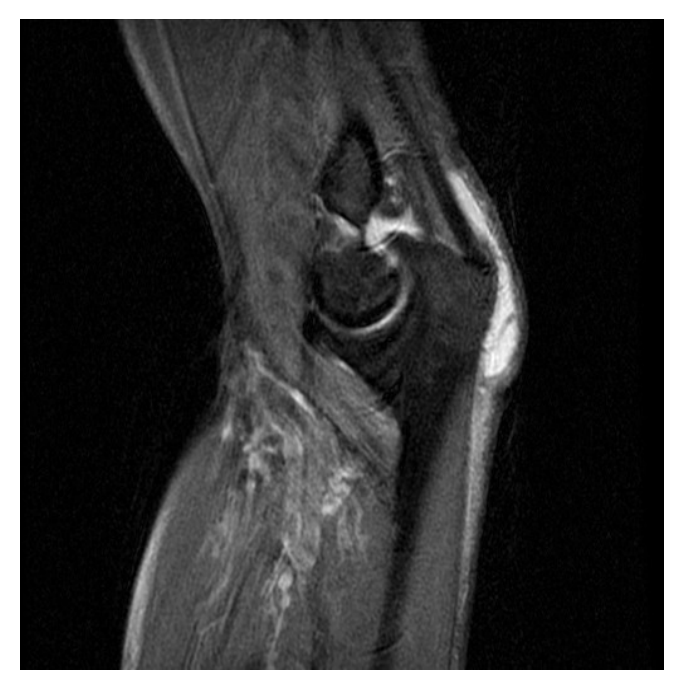

Figure 1b. Left Elbow MR PD Fsat Left elbow MRI imaging shows olecranon bursitis. a role in the aetiology. Our case did not have any systemic disease that can be lead to calcified lesions at periarticular soft tissue. Traumatic osteochondral lesions were detected on the head of radius supported that HO was secondary to trauma. The clinical finding of the patient was remained silent until developing acute bursitis. The development of $\mathrm{HOB}$ enabled the identification of pathology-induced trauma. As a result, there is overlooked a trauma and induced $\mathrm{HO}$ and $\mathrm{HOB}$ triggered by HO. The major trauma which resulted limb loss that the patient had led to overlook the trauma on the other side which affected relatively more less and HO induced by the trauma. This missed situation draws attention on the necessary of the evaluation of the asymptomatic limb in patients who had major trauma resulted with limb loss.

In the RSD treatment, medical therapy, physical therapy, sympathetic ganglion blocks and electro-stimulation techniques are being performed. Additional to physiotherapy, stellate ganglion block was performed three times to the case. Toshniwal et al. were found decrease in visual pain score at first week and was detected relief on oedema at fourth week of the stellate ganglion blockage [8].

\section{Conclusion}

This case report draws attention to the $\mathrm{HO}$ and $\mathrm{HOB}$ relationship will contribute to further work to be done in this regard.

\section{References}

1. Strickland RW, Vukelja SJ, Wohlgethan JR, Canoso JJ (2009) Hemorrhagic subcutaneous bursitis. J Rheumatol 18: 112-114. [Crossref]

2. Wasserman AR, Melville LD, Birkhahn RH (2009) Septic bursitis: a case report and primer for the emergency clinician. J Emerg Med 37: 269-272. [Crossref]

3. Kirou KA, Chaudhry N (2014) Olecranon Bursitis Is Often Hemorrhagic and Responds to Steroid Injections, Hospital for Special Surgery, New York, NY, Rheumatology, Hospital for Special Surgery, New York, NY, ACR/ARHP Annual Meeting.

4. Schumacher HR (1993) Arthrocentesis, synovial fluid analysis, and synovial biopsy In: Schumacher HR (Ed.), Primer on Rheumatic Diseases (10thedn), Atlanta, Ga: Arthritis Foundation, 67-72.

5. Mayadagli A, Bulut G, Ekici K (2012) Heterotopik Ossifikasyon ve Tedavi Yaklasimi J Kartal TR 23: 49-55.

6. Green SM (2000) Nonsteroidal anti-inflammatory drugs (NSAIDs). Tarascon Pocket Pharmacopoeia 2000. Loma Linda, Calif: J Rheumatol 18: 112-114.

7. Forouzanfar T, Köke AJ, van Kleef M, Weber WE (2002) Treatment of complex regional pain syndrome type I. Eur J Pain 6: 105-122. [Crossref]

8. Toshniwal G, Sunder R, Thomas R, Dureja GP (2012) Management of complex regional pain syndrome type $\mathrm{I}$ in upper extremity-evaluation of continuous stellate ganglion block and continuous infraclavicular brachial plexus block: a pilot study. Pain Med 13: 96-106. [Crossref]

Copyright: (C2017 Unlu Z. This is an open-access article distributed under the terms of the Creative Commons Attribution License, which permits unrestricted use, distribution, and reproduction in any medium, provided the original author and source are credited. 